\title{
Treatment of horizontal and vertical tidal signals in GPS data: A case study on a floating ice shelf
}

\author{
Matt King ${ }^{1}$, Richard Coleman ${ }^{1,2}$, and Peter Morgan ${ }^{3}$ \\ ${ }^{1}$ Centre for Spatial Information Science, University of Tasmania, GPO Box 252-76, Hobart, Tasmania 7001, Australia \\ ${ }^{2}$ CSIRO Marine Research, GPO Box 1538, Hobart, Tasmania 7001, Australia \\ ${ }^{3}$ School of Computing, University of Canberra, PO Box 1, Belconnen, ACT 2616, Australia
}

(Received December 20, 1999; Revised May 24, 2000; Accepted June 28, 2000)

\begin{abstract}
Knowledge of the surface velocity and strain of ice shelves is important in determining their present kinematic state and detecting any change in that state. Data collected using the Global Positioning System (GPS) often plays an important role in determing these parameters, either directly, or as ground-truthing to other techniques such as InSAR. The processing of GPS data on floating ice shelves is complicated by the presence of a distinct vertical tidal signal and large horizontal motions in the data. Over a one hour period, vertical and horizontal movements can be as much as 0.3 metres and 0.1 metres respectively. For such GPS data to be processed using conventional static methods would require the observation period to be split into small ( $\sim 1$ hour) segments, and the segments processed separately. Other processing options may include kinematic processing or sequential processing, although these techniques have their own drawbacks. Instead, we have developed software to remove signals based on a priori knowledge of the ice shelf motion. The tidal signal is removed using a local tide model and the horizontal velocity effect is corrected to a specific time epoch. This allows us to process our GPS data in a tide-free, velocity-free environment for a given day using conventional GPS processing software. The corrected GPS data, now largely free from the effects of ice shelf motion, may then be combined to produce high precision velocity and strain rate models of the ice shelf.
\end{abstract}

\section{Introduction}

Since the International Geophysical Year in 1957/58, Antarctica has been a significant focus of the international scientific community. In terms of glaciology, attempts to understand the dynamics of Antarctica's grounded continental ice sheet and floating ice shelves have led to somewhat extensive field campaigns in remote and hostile regions.

The original campaigns used conventional surveying techniques (i.e., astronomical, triangulation, trilateration and levelling observations) to measure position of ice movement markers at various epochs, normally separated by a period of months or years. These measurements were hampered by line-of-sight restrictions, and the inability to observe in poor weather conditions. More recently, Global Positioning System (GPS) measurements have been made in Antarctica, and these data largely overcome the types of limitations experienced in the previous surveys. Although, as outlined below, GPS technology also introduces difficulties of its own.

Ice shelves are of particular interest since they play a key role in two main areas: 1) 'buttressing' grounded continental ice, preventing rapid movement onto the ocean (Hughes, 1992), and 2) influencing local ocean water circulation (Wong et al., 1998). As a result, their break-up could cause significant changes to a large range of local, regional and global environmental parameters.

Copy right(c) The Society of Geomagnetism and Earth, Planetary and Space Sciences (SGEPSS); The Seismological Society of Japan; The Volcanological Society of Japan; The Geodetic Society of Japan; The Japanese Society for Planetary Sciences.
There are two major forces that act on an ice shelf, and changes in these forces may influence the likelihood of ice shelf break-up. Firstly, strain induced by the weight of the ice shelf causing it to thin and spread, and secondly, tidal action from underneath the ice shelf. GPS can be used to measure both of these parameters. When using GPS to measure positions or baselines on an ice shelf, the ice shelf's tidal motion is incompatible with the conventional static GPS processing model, and this motion needs to be either modelled or removed if high-precision distances (and hence strain) are to be calculated. The ice shelf's horizontal motion, although normally much less than the vertical motion during a 24 hour period, also conflicts with such a static model.

This paper deals with the horizontal and vertical tidal motions of the Amery Ice Shelf (AIS), East Antarctica (see Fig. 1), and shows how these motions can be removed from the raw GPS measurements prior to processing, allowing the GPS processing to proceed on an essentially tide-free, static surface. Corrected GPS data from different epochs may then be combined to reveal information, more precise than would have been possible without the removal of the signals, about the horizontal and vertical velocity and strain of the ice shelf.

\section{Ice Shelf Tidal Motion Measurements}

The vertical motion of an ice shelf induced by tides has been measured in a variety of ways: Levelling, tilt meters, gravity meters, bottom-mounted tide gauges, Electronic Distance Measurement (EDM), GPS, and recently Interferometric Synthetic Aperture Radar (InSAR) have all been used 


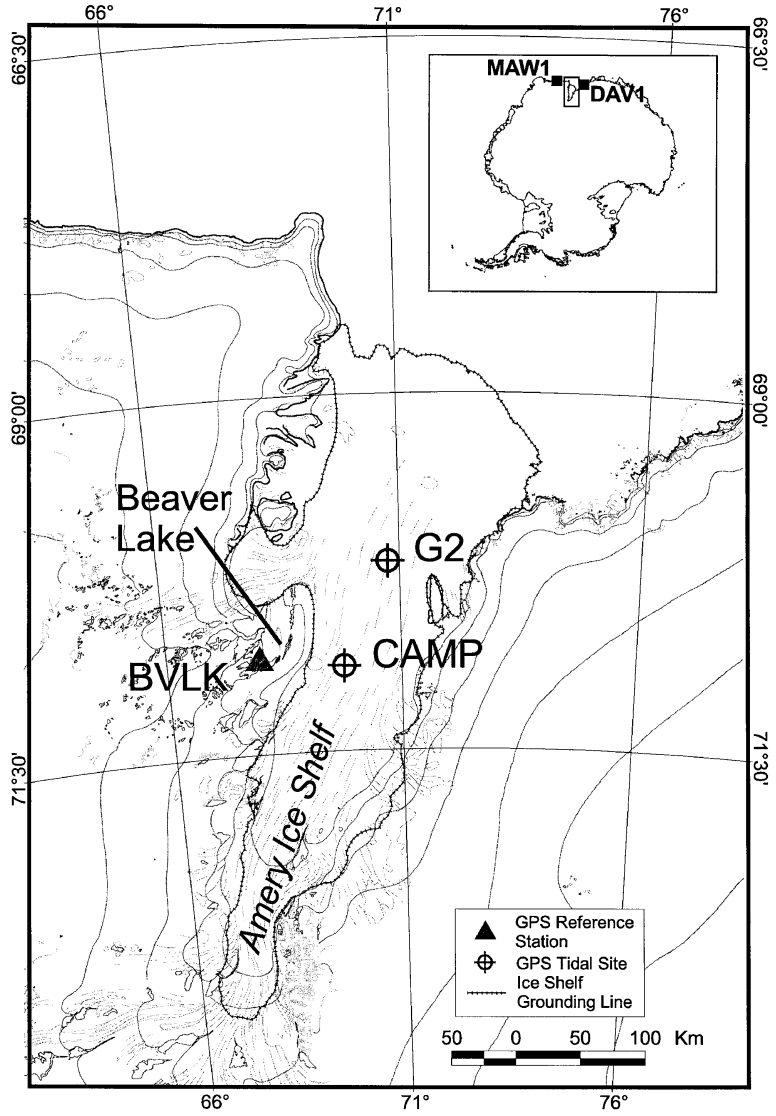

Fig. 1. Location of the Amery Ice Shelf and GPS measurement locations.

with varying degrees of success (e.g., Bondesan et al., 1994; Doake, 1992; MacAyeal, 1984; Pedley et al., 1986; Rignot et al., 2000; Stephenson et al., 1979; Vaughan, 1995). However, each method has certain limitations. While levelling, tilt meters, gravity meters and bottom-mounted tide gauges only provide measurements of vertical movement, EDM, GPS and InSAR also provide the potential for measuring horizontal motion as well. The later two techniques are able to resolve three-dimensional motion.

Both levelling and EDM rely on measurement areas being close to non-tidal regions (so as to establish a datum) and good visibility, a restriction that limits the effectiveness of these methods on large Antarctic ice shelves, such as the AIS. Tilt meters work well in ice shelf grounding zones, where tilt is larger, but are less effective further afield. Solid monumentation of the tilt meter in the ice is the main difficulty of this technique. Gravity meters provide measurements of the gravity changes as water moves under the ice shelf, and has been shown to work effectively with results agreeing well with independent tide models. Bottom-mounted tide gauges have been used successfully on the George VI ice shelf (Pedley et al., 1986), although they are both difficult and expensive to install and retrieve, making them impractical in many situations.

The InSAR technique has been used to accurately identify the grounding zone of large regions of ice shelves, and more recently to identify constituents of tidal motion. This method is presently somewhat limited since the orbital periods of the current satellites are in phase with semi-diurnal tidal motion. As a result, long time periods are required to resolve the majority of constituents (Rignot et al., 2000). There may be several months between satellite passes, and since basal melt/freeze or surface melt/accumulation/ablation will cause an ice shelf to adjust vertically, potential systematic errors exist if these parameters are not known. Future SAR satellites may have different orbital periods and hence not face this limitation.

GPS data have been used to detect and define ice shelf grounding zones using kinematic processing, while these data have also been used for vertical measurements away from grounding zones using both kinematic and static techniques. In our experience, kinematic data processing over baselines longer than $20 \mathrm{~km}$ suffers from the high residual ionospheric noise present in the data, especially in the AIS region (and over most of Antarctica). Processing GPS data on an ice shelf using a static methodology also poses difficulties.

While the ice shelf motion can be measured by GPS, what is needed for integration of such measurements is a static reference system on the ice shelf. The advantage of such a static reference system is that it allows the integration or comparison of long-term horizontal or vertical positions or velocities in a consistent reference frame. For the vertical component, this is best provided by a tide-free surface, such as a specified datum level (treatment of the other geophysical corrections, such as solid earth tides, is also required and is dealt with later), whilst for the horizontal components, we need to correct all data to a specific epoch during the measurement period. Implicit in the above is the assumption that the motion of the ice shelf over some time period can be exactly described by some chosen model, such as a tide model or a horizontal velocity field. Any imperfections in the models used or differences between this model and the GPS data will directly cause biased results. With these stated assumptions, we describe below procedures for removing ice shelf motion from raw GPS observations.

\section{Initial Data Processing}

GPS data collected on the AIS over three Antarctic field seasons (1995/96, 1997/98, 1998/99) have previously been processed by King et al. (2000), using two different static data processing methodologies. In this paper we adopt the first of these methods, to determine, as a first step, the phase difference between GPS data located on the AIS and an independant tide model generated from tide gauge data at Beaver Lake (see Fig. 1). We also determine short-term horizontal velocities at this stage. In this method, the GPS data is segmented and each segment is regarded as being static for its entire period, and processed as normal using GAMIT software (King and Bock, 1999). The findings of King et al. (2000) are summarised below:

- the vertical signals at different locations agreed well with the Beaver Lake tide model after accounting for phase differences

- an amplitude change was apparent in GPS results when compared to the tide model 


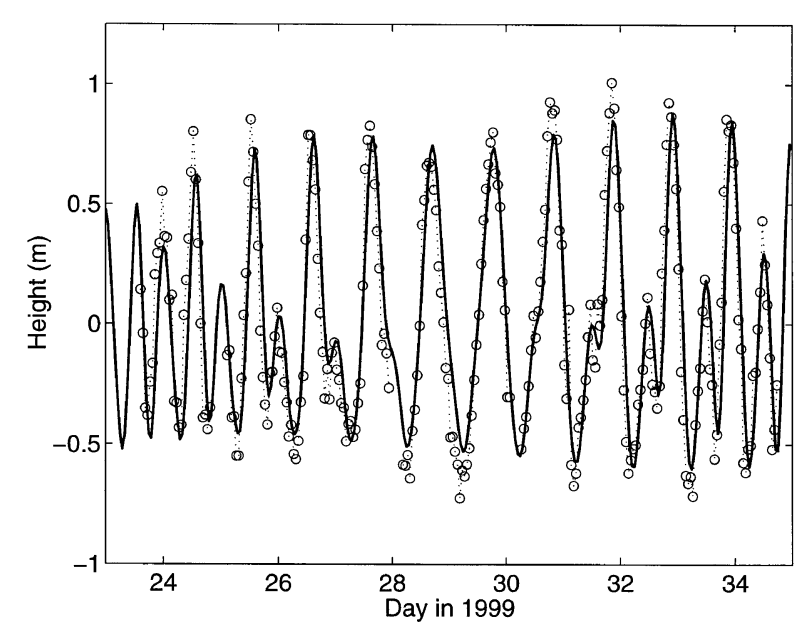

Fig. 2. The agreement between hourly GPS positions (circles and dotted line) at and the independent Beaver Lake tide model (solid line; taken from King et al., 2000). No phase shift has been applied to the tide model, in this case, although the difference is approximately 45 minutes. A mean offset has been removed from each of the data sets.

- a phase gradient was evident along the length of the ice shelf

- calculated short-term horizontal velocities (over a few days) compared well with long-term results (over three years).

Figure 2 shows an example of the agreement of GPS data with the Beaver Lake tide model using this technique. Formal errors (one sigma) for the GPS data are approximately $5 \mathrm{~cm}$.

\section{Removal of the Vertical Motion}

Since the Beaver Lake tidal model provides a good comparison to the GPS data in this region, we use it as a representative of a true ice shelf response to the tides, and any difference between it and the GPS results is regarded as measurement error. In regions where tide gauge observations have not been made, or are not possible, predictions based on long-term (preferably more than 1 month) GPS observations or a regional tidal model (e.g., Robertson et al., 1998) could be used. In order to remove the tidal signal from the GPS data, we use a five step process.

1) Solve for a best-fit phase shift of the tide model to the GPS data, using a cross correlation technique. Computed shifts for the AIS are presented in King et al. (2000) and are approximately 45 minutes, with the GPS sites on the ice shelf leading (i.e., motions occurring before) the tide model.

2) Apply this shift to the tide model and compute the modelled tidal value at each GPS epoch of measurement.

3) Read in raw GPS data from a RINEX observation file and convert the carrier phase ranges to units of metres.

4) Read in a RINEX navigation file for calculating the position (and hence elevation) of satellites at the measurement epochs. Earth rotation during signal travel time is also accounted for at this stage.

5) Correct the observed ranges as shown below and write out a new RINEX file.

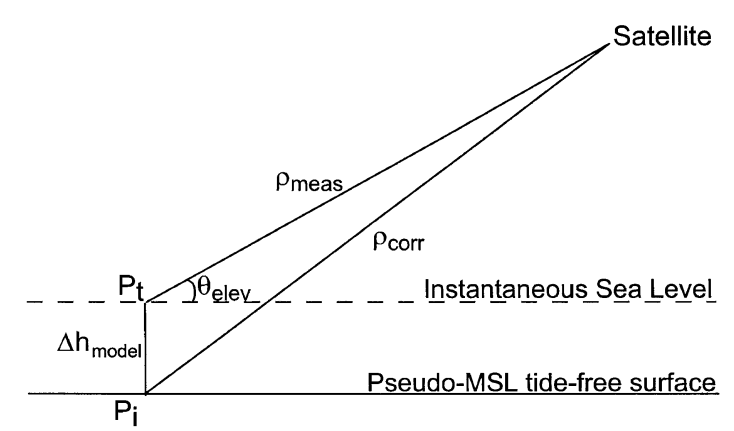

Fig. 3. The geometry (two-dimensional) involved in removing a vertical motion from a GPS range.

The geometry of the problem is two-dimensional and is presented in Fig. 3. Using the cosine rule, the tide-free corrected range for a particular satellite is (in metres):

$$
\begin{aligned}
\rho(t)_{\text {corr }}= & {\left[\rho(t)_{\text {meas }}^{2}+\Delta h(t-\delta)_{\text {model }}^{2}\right.} \\
& -2 \rho(t)_{\text {meas }} \Delta h(t-\delta)_{\text {model }} \\
& \left.\cdot \cos \left(90^{\circ}+\theta(t)\right)\right]^{\frac{1}{2}}
\end{aligned}
$$

where:

$\rho$ is the corrected and measured carrier phases (converted to metres) and pseudoranges

$\Delta h$ is the instantaneous modelled height of the tide above a pseudo-MSL tide-free surface

$\theta$ is the angle of elevation of the satellite above the horizon

$t$ is the time at some measurement epoch

$\delta$ is the computed phase shift required to fit the tide model to the GPS data.

The corrected ranges may then be converted back into carrier phase cycles where necessary, and written back into a RINEX file. Note that this correction is satellite azimuth independent, and only relies on the elevation angle of the satellite.

\section{Removal of the Horizontal Motion}

In order to remove the horizontal motion of a GPS receiver during an observation session, an estimate of the horizontal velocity of the receiver is required. In practice these could be obtained via long-term GPS observations, satellite techniques or, as we have done in this paper, short-term GPS observations (King et al., 2000).

The correction of receiver-satellite ranges for horizontal motion of the GPS receiver is more complicated than the correction for vertical motion, since the correction is dependent on the azimuth of the satellite. The geometry of the problem is three-dimensional and is shown in Fig. 4. Using two applications of the cosine rule and taking advantage of similar triangles, the horizontal velocity-free corrected range for a particular satellite is (in metres):

$$
\begin{aligned}
\rho(t)_{\text {corr }}= & {\left[\rho(t)_{\text {meas }}^{2}+(b(t)-a(t))^{2}\right.} \\
& \left.-2 \rho(t)_{\text {meas }}(a(t)-b(t)) \cos \left(E l_{1}(t)\right)\right]^{\frac{1}{2}}
\end{aligned}
$$

where:

$$
a(t)=\rho(t)_{\text {meas }} \cos \left(E l_{1}(t)\right)
$$




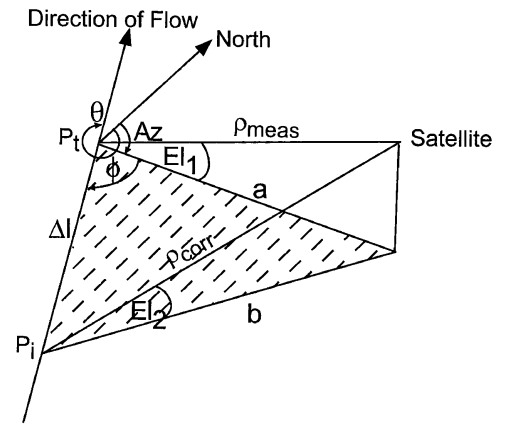

Fig. 4. The geometry (three-dimensional) involved in removing a horizontal velocity from a GPS range.

$b(t)^{2}=a(t)^{2}+\Delta l(t)^{2}-2 a(t) \Delta l(t) \cos \phi(t)$

$\phi(t)=\left(A z(t)-\theta+180^{\circ}\right)$, if $t>i$ (measurement occurs after the reference epoch), or otherwise, $\phi(t)=(A z(t)-\theta)$

$A z(t)$ is the observed azimuth of the satellite

$E l_{1}(t)$ is the observed elevation of the satellite

$\theta$ is the azimuth of ice flow

$\Delta l(t)$ is the horizontal distance the ice travels along azimuth $\theta$ during time $t-i$

$i$ is the time of the chosen reference epoch.

Using this approach the GPS signal can be corrected to any given reference epoch, however since most static GPS processing software relates position to $12: 00$ on the day of observation, in our processing we always correct the ranges to a midday epoch $(i=43200 \mathrm{~s})$.

It is noted that Eqs. (1) and (2) are analogous to the corrections applied when correcting GPS observations for the phase-centre variations of a GPS antenna.

\section{Analysis of Results}

\subsection{Case 1}

Both the tide-free and velocity free corrections were applied to a 34 hour data set at station G2 located on the AIS (see Fig. 1). The phase-shifted Beaver Lake tide model was used for the vertical corrections and the short-term velocities given in King et al. (2000) used for the horizontal corrections. For the purpose of comparison with the original segmented results, we processed the corrected data in hourly segments and show the results in Fig. 5. Both the uncorrected and corrected segmented GPS data were processed in GAMIT software in a 3-4 station regional network. Sites DAV1 and MAW1 (see Fig. 1) were tightly constrained (within $10 \mathrm{~mm}$ ) to their ITRF96 values while loosely constraining the remainder of the sites (within $100 \mathrm{~m}$ ). Scripps Orbit and Permanent Array Center (SOPAC) precise ephemerides were held fixed, while using an satellite elevation cut-off angle of $15^{\circ}$. Corrections were made for frequency dependent and solid earth tides. Thirteen tropospheric parameters per site per day were estimated, and elevation-dependent phase centre models were implemented as provided by the IGS (see for e.g., Mader and Mackay, 1996) for different GPS antenna types (all sites in this paper were occupied with choke-ring antennas, except for G2 where a Leica LC202G antenna was installed).

Results from the GAMIT solutions were transformed into a local North-East-Up system and are shown in Fig. 5. The
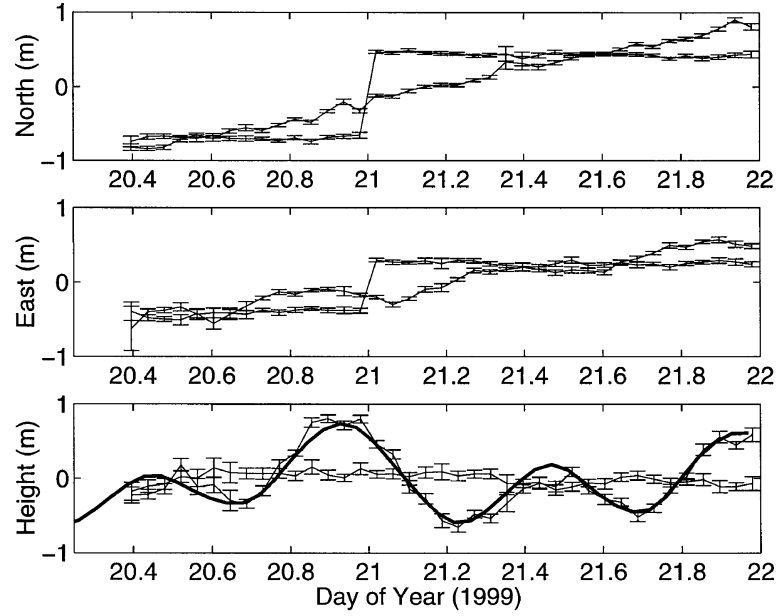

Fig. 5. One hour solutions of the original data set and the tide and velocity-free data set at site G2. The bold line in the height component is the phase-shifted Beaver Lake tide model. A mean offset has been removed from each of the data sets.

error bars are one sigma formal errors from the processing software, and are approximately $5 \mathrm{~cm}$. The motions in the East-West component of the uncorrected data will not be discussed further here as they are the subject of a more detailed investigation to be reported elsewhere. The jumps at day 21 in the North and East components of the corrected data sets are due to the choice of midday on the day of observation as the reference epoch.

With regard to the vertical correction, it can be seen that the tidal signal is largely removed. The standard deviations of the corrected solution are $9 \mathrm{~cm}$ for day 20 and $7 \mathrm{~cm}$ for day 21 in the vertical component. In terms of the horizontal correction, the results are even better, with standard deviations being 2.7 $\mathrm{cm}$ (day 20, 21) in north, and $6.6 \mathrm{~cm}$ (day 20) and $2.9 \mathrm{~cm}$ (day 21) in east. After removing the first point from the data set the standard deviation for the east component on day 20 became $2.8 \mathrm{~cm}$. No other significant signs of misfit are evident, showing that the motion of the antenna was constant throughout the day.

\subsection{Case 2}

In order to test the performance of the 'static' ice site we have processed 10 days of corrected GPS data from site CAMP (see Fig. 1) on the AIS, this time without segmenting the data (i.e., CAMP has one 24 hour segment per day). The GPS processing was performed as described above, although a larger ( $\sim 20$ site) regional network was used. We compare the formal errors (from the GAMIT software) of CAMP with the formal errors from the same solution of nearby rock site BVLK (see Fig. 1). Identical receivers were present at both locations, and therefore receiver noise should be similar at both sites. Since formal errors presented in GPS processing software are a measure of the internal measurement noise, this test should reveal the quality of the corrected 'static' data. The 10 day time series of formal errors at both sites are presented in Fig. 6 in the form of a Box-and-Whisker plot.

Over the length of the campaign, the mean formal error for BVLK (rock site) was better than that of CAMP (the ice shelf site) in all three components; by $1.1 \mathrm{~mm}$ in north, $1.3 \mathrm{~mm}$ in 


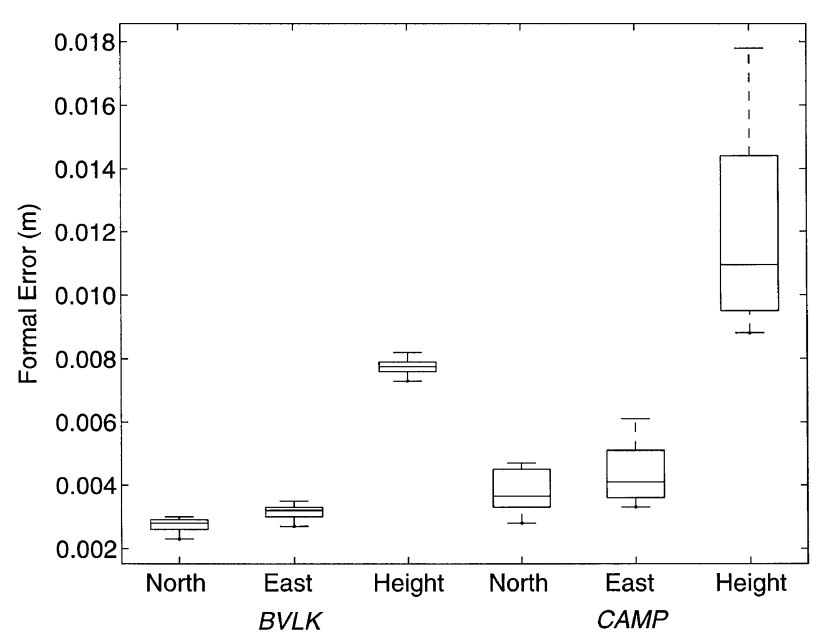

Fig. 6. Box and Whisker plot of the formal errors from GAMIT of each component at sites BVLK and CAMP over 10 days.

east and $4.6 \mathrm{~mm}$ in height. The formal errors of the CAMP site were also more variable than the BVLK site. Overall, this shows that while there is some residual noise present in the CAMP data, the velocity and tide-free GPS data does appear to conform well to the static GPS processing model.

\section{Conclusions}

It has been shown that given appropriate a priori information, horizontal and vertical tidal motion can be removed from raw GPS data collected on floating ice shelves. The resulting GPS observations differ only slightly in their precision from those observed on a stable surface. As a result, these tide and velocity-free data may then be processed using a normal static GPS processing package without need for segmentation, thereby increasing the precision of the results. Measurements conducted over several days or from different campaigns may then be compared or combined to produce results of a higher precision than would have been possible otherwise. Changes in the state of the ice shelf may then be detected over shorter time periods than would have been possible otherwise. On the Amery Ice Shelf, these high precision positions and baselines will make a significant contribution towards our understanding of the strain fields in this highly dynamic region, as well as provide valuable ground-truthing for current and future satellite missions.

Acknowledgments. The authors would like to thank Gilbert Strang and Kai Borre for making available their collection of MATLAB $\mathrm{m}$-files, which were used extensively in the software described in this paper. Tidal Constituents for Beaver Lake were supplied by the National Tidal Facility, The Flinders University of South Australia, Copyright reserved. Paul Tregoning provided the BVLK GPS data. Comments by Kazuo Shibuya and Eric Rignot helped in clarifying the contents of this paper. This work is supported by ASAC and ARC grants.

\section{References}

Bondesan, A., A. Capra, A. Gubellini, and J. Tison, On the use of static GPS measurements to record the tidal response of a small Antarctic ice shelf (Hells Gate Ice Shelf, Victoria Land), Geografia Fisica Dinamica Quaternaria, 17, 123-129, 1994.

Doake, C. S. M., Gravimetric tidal measurements on Filchner Ronne Ice Shelf, in 7th International Workshop of the Filchner-Ronne Ice Shelf Programme, edited by H. Oerter, pp. 34-39, Alfred-Wegener-Institute for Polar and Marine Research, SchloB Senden, Germany, 1992.

Hughes, T., On the pulling power of ice streams, Journal of Glaciology, 38(128), 125-151, 1992.

King, R. W. and Y. Bock, Documentation for the GAMIT GPS analysis software (version 9.8), Unpublished, Massachusetts Institute of Technology, 1999.

King, M., L. Nguyen, R. Coleman, and P. Morgan, Strategies for high precision processing of GPS measurements with application to the Amery Ice Shelf, East Antarctica, GPS Solutions, 4(1), 2-12, 2000.

MacAyeal, D. R., Numerical simulations of the Ross Sea tides, J. Geophys. Res., 89(C1), 607-615, 1984.

Mader, G. L. and J. R. Mackay, Calibration of GPS antennas, in 1996 Analysis Center Workshop, edited by R. E. Neilan, P. A. Van Scoy, and J. F. Zumberge, pp. 81-105, IGS Central Bureau, Silver Spring, Maryland, 1996.

Pedley, M., J. G. Paren, and J. R. Potter, The tidal spectrum underneath Antarctic ice shelves, J. Geophys. Res., 91(C11), 13001-13009, 1986.

Rignot, E., L. Padman, D. R. MacAyeal, and M. Schmeltz, Observation of ocean tides below the Filchner and Ronne Ice Shelves, Antarctica, using synthetic aperture radar: comparison with tide model predictions, J. Geophys. Res., 105(C8), 19615-19630, 2000.

Robertson, R. A., L. Padman, and G. D. Egbert, Ocean, Ice and Atmosphere, Interactions at the Antarctic Continental Margin, Vol. 75, edited by S. S. Jacobs and R. F. Weiss, pp. 341-369, AGU, Washington, 1998.

Stephenson, S. N., C. S. M. Doake, and J. A. C. Horsfall, Tidal flexure of ice shelves measured by tiltmeter, Nature, 282, 496-497, 1979.

Vaughan, D., Tidal flexure at ice shelf margins, J. Geophys. Res., 100(B4), 6213-6224, 1995.

Wong, A. P. S., N. L. Bindoff, and A. Forbes, Ocean, Ice and Atmosphere, Interactions at the Antarctic Continental Margin, Vol. 75, edited by S. S. Jacobs and R. F. Weiss, pp. 173-187, AGU, Washington, 1998.

M. King (e-mail: ma_king@postoffice.utas.edu.au), R. Coleman, and P. Morgan 